\title{
The Role of Collaborative Networks in Sustainability
}

\author{
Luis M. Camarinha-Matos ${ }^{1}$, Hamideh Afsarmanesh ${ }^{2}$, and Xavier Boucher ${ }^{3}$ \\ ${ }^{1}$ Universidade Nova de Lisboa, Faculty of Sciences and Technology, Portugal \\ cam@uninova.pt \\ ${ }^{2}$ University of Amsterdam, Netherlands \\ h. afsarmanesh@uva.nl \\ ${ }^{3}$ Ecole Nationale Supérieure des Mines de St Etienne, France \\ boucher@emse.fr
}

\begin{abstract}
There is a great potential for mutual beneficial synergies between the two fields of Collaborative Networks and Sustainability science. This potential has in fact been recognized in many fields where these synergies are leading to novel approaches and solutions. It is particularly evident that the challenges of sustainability call for a wide collaboration among multiple stakeholders, as the needed changes exceed the capacity and capability of individual actors. With the purpose to illustrate the possible contribution and challenges for collaborative networks in sustainability, a number of relevant cases where examples of such synergies are already identified are briefly introduced.
\end{abstract}

Keywords: Collaborative Networks, Sustainability, Stability.

\section{Introduction}

The notion of sustainability is a subject of growing importance. It is present in most strategic and political agendas, being a prevalent issue in science and technology, and leading to related terms such as sustainable development [1] or even sustainability science. One important milestone was the UN's World Commission on Environment and Development report of 1987 [2], also known as the Brundtland report, which defined sustainability as:

"Meeting the needs of the present without compromising the ability of future generations to meet their own needs."

Environmental and ecological aspects have dominated the discussions on sustainability, which is natural since after all the environment is the elementary basis of life. Nevertheless, such perspective falls short if we do not pay attention to other aspects such as resource management, organizational forms of the society, cultural aspects, etc. Therefore, more recent discussions, as represented in the conclusions of the 2005 World Summit [3], [4], led to the identification of three main pillars of sustainability (Fig. 1):

- Environmental - aiming at maintaining a stable base of resources, preserving ecosystems and biodiversity, avoiding over-exploitation of renewable resources, safeguarding the quality of the atmosphere, recycling, etc. 
- Economical - focusing on the ability to generate wealth in the long term, namely producing goods and services, creating jobs and prosperity, pursuing efficiency, etc.

- Social - concerned with achieving equity, providing social services, guaranteeing social inclusion, preserving cultures, groups, places, and ensuring political accountability and participation (community).
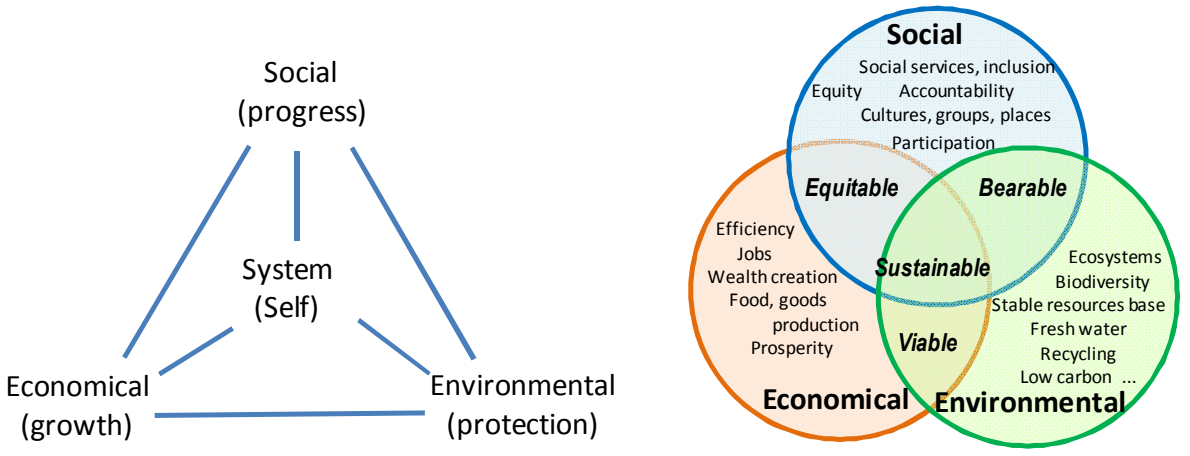

Fig. 1. Pillars of sustainability

Clearly, all these elements of sustainability, although an incomplete list of examples, introduce considerable complexity. Not only the goals expressed are clearly multi-dimensional but, additionally, they have to be coordinated within a long term vision of performance and development. Such complexity raises the issue of how to balance (sometimes incompatible) objectives and also how to measure the achievement level. Moreover, it is noticeable that new key actors, including the private sector, are now playing an increasingly constructive role. Due to the growing awareness among political sphere and in the civil society of the importance of sustainability, also the business sector starts to acknowledge that preserving the environment and the other inter-related pillars is both good business and a moral obligation. More strict regulations, consumers' demand for greener products, reduction of the carbon footprint, optimization of the usage of natural resources, more societal attention, and many other trends are triggers for the emergence of green, environmental-conscious, as well as social-oriented markets. At the academic and research level, the challenges of sustainability are becoming a key topic for various scientific communities and in some universities new focused units are being created.

Sustainability challenges clearly call for a wider collaboration as the needed changes exceed the capacity and capability of individual actors. The discipline of Collaborative Networks has certainly a key role to play, in the multi-stakeholder collaborative perspective which has to be now implemented.

\section{Why Collaboration}

Concepts, methods and tools developed in the area of Collaborative Networks (CNs) can facilitate the engagement and interplay of the multiple stakeholders which are participants needed in any effort towards sustainability. For instance: 
- Basic infrastructures and collaboration tools - support interaction, information sharing and exchange (a basis for informed decision-making), coordination of potentially heterogeneous, distributed and autonomous entities.

- The notion of community, as implicit in the Virtual organization Breeding Environments (VBEs) or Professional Virtual Communities (PVCs), helps in building the perception of mutual dependency and co-responsibility for the stakeholders involved.

- What we have learned with studies on trust management, value systems, multicultural and multi-legal contexts for CNs can help in the development of better policies and governance methods for sustainable development projects. Sustainability is a global challenge and therefore it needs to be addressed at a planetary scale and with a multidimensional approach.

On the other hand, conceptual developments in CNs will also benefit as we gather deeper knowledge on the mechanisms involved in sustainability. One example is given by the emerging discipline of biomimicry or biomimetics, which studies Nature's best "ideas" in terms of models, systems, processes, and elements, and tries to imitate these designs and processes to solve human problems. The core assumption is that Nature has already solved many of the problems we are facing. This idea is also reflected in expressions such as "innovation inspired by nature" and has been, in fact, adopted by the collaborative networks research community. For instance, the notion of a business ecosystem, typically seen as a particular case of VBE, is an example of biomimicry, as it gets its inspiration from natural ecosystems.

But this analogy can be taken further. An ecosystem does not involve only collaboration but rather a complex interplay of collaboration and competition, producers, consumers, regulators, and support entities. Although a multiplicity of roles and even competition are present, there is some form of "survival instinct" and even shared vision that enables participants to align their investments and to find mutually supportive roles. Exploring these ideas will bring new insights on better governance principles that will likely help in reducing the percentage of failures and thus contributing to more sustainable business ecosystems. Even though lacking proper formalization, ideas such as business or organizational ecologies also touch on this issue. Other examples come from the so-called "bio-inspired" algorithms, e.g. swarm intelligence and genetic algorithms, which are progressively exploited to solve issues of self-organizing systems, distributed systems design, optimization and distributed scheduling, etc. In addition, sustainable development is not a fixed state of harmony, but rather a process of change in which the exploitation of resources, direction of investments, orientation of technological development and institutional changes need to be made consistent with present as well as future needs. This notion can also influence further research emphasis on dynamic models, networks evolution, and network-health supervision, which are subjects not yet well developed in CNs.

Therefore there is a great potential for mutual beneficial synergies between the two fields of Collaborative Networks and Sustainability Science. The following sections briefly introduce a number of relevant cases where examples of such synergies have already been identified. 


\section{Business Ecosystems}

This is perhaps one of the most developed areas in Collaborative Networks. The advances in Information and Communication Technologies (ICT), especially the Internet and pervasive computing, have revolutionized virtual collaborations and enabled or induced the emergence of new paradigms such as extended enterprise, virtual enterprise (VE) / virtual organization (VO), and professional virtual community. All of these concepts represent the temporary alliances of entities that come together to share their skills, core competencies and resources in order to better respond to emerging business opportunities, and whose collaboration is supported by computer networks. In practice however, the rapid formation of a consortium to respond to a business opportunity, which is a major "selling point" for the VE/VO paradigm, faces many obstacles: notably the large heterogeneity of the autonomous participants involved (in terms of their technological infrastructures, business practices, culture, etc.), as well as the time needed to build trust. As a consequence, the concept of VO Breeding Environment (VBE) has emerged. A VBE is a long term association which aims at increasing the level of anticipatory preparedness of its members. This enables their more efficient integration and participation in temporary alliances, as triggered by emerging business opportunities [5]. This concept is a successor of earlier organizational structures such as industry clusters or industrial districts, but with better ICT support and more adequate governance models.

A business ecosystem, also known as digital business ecosystem, can be seen as a particular case of a VBE with, as its name implies, principles and mechanisms inspired from biological ecosystems. These ecosystems are frequently focused on one geographical region and try to involve the socio-economic living forces of that region. The industry cluster is one of the earliest forms of business ecosystems, consisting of a group of companies, typically located in the same geographic region and operating in a common business sector. Its function is to keep some long-term "binds" with each other in order to increase their general competitiveness in the larger region. These binds or agreements may include sharing some buyer-supplier relationships, common technologies and tools, common buyers, distribution channels or common labor pools, all contributing to some form of cooperation or collaboration when business opportunities arise. Earlier forms of clusters did not require a strong ICT infrastructure but more and more often, today's collaboration requires such support. The term industrial district, mostly used in Italy, represents a concept quite similar to an industry cluster. It can be focused on one single sector or cover a number of sectors in a given region. Another organizational structure that shares some characteristics with the above examples is the case of enterprise incubators. An incubator represents a pool of small companies in their early phase, co-located in the same geographical space, possibly covering different sectors. These start-ups share some basic infrastructures (communications and other generic services) as well as consultancy support in order to evolve towards mature organizations. However, traditional incubators are not yet real VBEs as they usually do not collaborate much in joint business opportunities. Nevertheless, it would be reasonable to imagine a next generation of incubators "absorbing" the goals, principles and mechanisms of a sustainability oriented VBE. 
Besides the production and services industries, these organizational structures and related concepts are being adopted, although some times with different terminologies, in a growing variety of other domains such as: collaborative e-governments, education networks, remote assistance networks for the elderly, collaborative escience and virtual laboratories, integrated transportation systems or environmental and disaster management systems, among many others. Related notions can also be found in some developments within the service oriented systems, such as the service parks and service ecologies.

\section{Collaborative Agribusiness}

Sustainable agriculture is understood as a way of raising food that is healthy for consumers and animals, does not harm the environment, is humane for workers, respects animals, provides a fair wage to the farmer, and supports and enhances rural communities. It thus addresses a number of key issues:

- Conservation and preservation of the environment,

- Support of biodiversity,

- Guarantee of animal welfare,

- Economic viability for farmers,

- Promotion of social justice for workers.

During the last decade the agribusiness industry has been forced to go through a major restructuring. The main driver has been economic, as a result of globalization and technological innovation. In addition, increased customer quality requirements, new environmental regulations, and the growing importance of "biological products", are playing a role in this process.

In order to survive, small producers need access to markets and to comply with increasingly tough regulations. This requires them to join efforts, e.g. by the formation of regional agribusiness clusters, where CNs can contribute. Needless to say, the survival of small producers is a fundamental element for the preservation of the culture and social cohesiveness in many regions. A survey of experiences in this direction in various European countries can be found in [6]. However, as for the agricultural and food industry, this shift towards virtual enterprises is somewhat under-developed. Nevertheless to the companies involved in food processing industry, it is becoming clear that a real competitive advantage can only be achieved and sustained through the creation of relationships, strong information links, and coworking among the enterprises along the various steps of the value chain. In our view, the creation of virtual enterprises is inevitable to efficiently utilize all of the relevant human, organizational, and business resources and to facilitate the necessary interdependencies between the suppliers (farmers and others), manufacturers, distributors, sellers as well as ultimately customers and consumer associations.

An interesting example of a collaborative network of agrifood SMEs and their client groups can be found in the Sibary region (Italy) as studied in [7]. Also in the production of canned tomato paste, enterprises which might work together to constitute a virtual enterprise include wholesalers and retailers, can producers and their supply chains, food processors and packers, farmers and fruit growers, 
transportation and delivery enterprises [8]. Such collaborative network would cover the set of all operations involved in the Agribusiness concept: production operations within the farm and storing, processing and distribution of all products and their derivatives. New regulations regarding traceability of food products also require new processes for information sharing and exchange. This again increases the need for greater communication and coordination among stakeholders involved in value-added partnerships.

In the same direction, the FORAGRO Position 2010 [9] identifies a number of mechanisms, e.g.:

- "To promote a better integration between the public and the private sectors at all levels, as well as articulate public-private networks to make progress in the development of policies for innovation;

- To formalize alliances and mechanisms for the exchange of information and experiences;

- To promote mechanisms to facilitate access to new markets and decrease the risk for small- and family-scale agriculture;

- To strengthen consumer organizations and their participation in RDI processes;

- To agree on regulatory frameworks and others for the management of shared resources (water, basins, biodiversity, soils);

- Etc. “

These could be greatly facilitated by a collaborative networks approach.

\section{Collaborative Transportation Systems}

The transportation / mobility sector is one of the most critical elements in sustainability, especially from the environmental and economic perspectives. Current development trends towards intelligent transport systems are leading to the progressive introduction of new technologies based on electronics, sensorial systems, and communication and information technologies. For instance, new road management policies, based on new user-paying models, and increasing concerns about traffic safety, establish requirements for a new family of emergent business services. A promising strategy to promote sustainable and safe mobility aims at offering new comprehensive service contracts integrating multiple possibilities of access to public transport systems, parking areas, subscription to innovative insurance policies, etc....

The next challenge is the development of a collaborative eco-driving environment, focused on effective support of integrated services targeting transportation energy efficiency, costs saving, and improvement of safety in mobility across Europe. There is an enormous potential for reducing accidents, optimizing mobility time, and improving energy efficiency. This requires enabling the vehicles and road infrastructures with a new generation of intelligent transportation systems. Furthermore, achieving such infrastructure on a large scale needs the currently fragmented scenarios of multiple stakeholders acting independently (and even in competition) to disappear and be replaced with a collaborative context promoting integrated services and service innovation. 
The underlying assumption is that new business models and novel business entities will progressively emerge in the transportation domain. In the future eco-driving environment, Integrated Transportation Service Providers will act either as service brokers or integrators directly interacting with the end-users, providing them with a range of integrated services. All the while, the actual service providers and their transactions will become invisible for the individual drivers. Through a single contract, the driver is released from the burden details of dealing with separate business entities and, additionally, gains an advantage in reduced cost via subscribing to several services at once. Furthermore, providers incur lower service and administrative costs. The actual composition of elemental services into more complex (integrated) service-packs is built dynamically and depends only on the subscription options taken by each driver. For instance, one customer might be interested in the service for optimal routing and optimal parking, as well as navigational information. Such services are likely to involve a number of different actors, such as road and highway operators, city traffic controllers, parking lots and gas stations owners, payment clearance operators, etc., for which significant interoperability is required.

Some successful "specialized" examples have already started to emerge on the market, although still with much of an ad-hoc nature. Take for instance the case of the "Via-Verde" in Portugal. This integrated service offer extends the traditional electronic tolling service of motorways with covering the parking services, gas station payments, food in drive-in restaurants, etc. [10]. The growing success of this initiative clearly shows the potential for integrated transportation-related services, supported by a collaborative network of service providers. While challenging, it is therefore important to leverage these initial ideas through provision of systematic and comprehensive models and dedicated infrastructures. These will not only facilitate the addition of new services but also provide conditions and support for authorities to supervise, influence and improve the general mobility and transportation throughout their regions and areas, thus saving energy and costs as well as improving safety.

\section{Smart (Energy) Grid}

The so-called smart grid represents a move from a centralized, energy producercontrolled network to one less centralized and more consumer-interactive. Initially, it corresponds to an overlay of the energy distribution grid with an information and metering system. At the current stage, most efforts are very focused on infrastructure aspects [11], [12]. However, establishing a truly smart grid requires the participation of a large number of stakeholders, including producers, transmission and distribution operators, regulators, policymakers, and consumers. Thus, the next challenge is to adopt organizational models, governance structures, and develop advanced tools to support collaboration among these players.

A brief analysis of the main (desirable) features of a smart grid clearly shows the potential for CNs in this domain:

- Self-healing from power disturbance events - initially focused on sensors, acquisition of real-time information, monitoring, error detection, diagnosis and recovery, that is a typical classical intelligent supervision problem. When the 
scope becomes grid-wide we have to deal with a network of autonomous entities and the approach needs to be one of collaborative systems.

- Enabling active participation of consumers - in its essence, a smart grid attempts to change consumers' behavior via variable electric rates or to pay vastly increased rates for the privilege of reliable electrical service during highdemand. These grids include consumer equipments in an overall strategy of consumption optimization and are also related to intelligent buildings. The notion of shared responsibility implies that consumers be treated as partners, namely in terms of benefit sharing, and not just as simple customers.

- Operating resiliently against physical and cyber attack - as systems become more complex and ICT-based, resilient architectures design can benefit from a collaborative networks approach.

- Providing higher power quality - smart grids are expected to help achieving more stable power supply. One of the elements involved here is proper coordination of the various producers and distribution subsystems, which given the diversity and autonomy of such entities, requires more than a traditional control approach.

- Accommodating all generation and storage options - progressive addition of renewable energy sources, including micro-generation (e.g. solar panels at home), new energy harvesting methods, and options for energy storage, lead to a large pool of resources whose management can benefit from the resource sharing and management results from collaborative networks.

- Enabling new products, services, and markets - a variety of new services and more dynamic energy markets are likely to emerge, which also require proper treatment of issues being addressed by the $\mathrm{CN}$ community such as distribution of benefits, negotiation protocols and e-contracting, privacy and security.

- Optimizing assets and operating efficiently - better harmonization of local distribution with interregional energy flows, smooth integration of renewable energy resources, which are for the most part intermittent in nature, betterinformed forecasting approaches, etc., are directions that could also benefit from a collaborative networks perspective.

One of the first works advocating the adoption of the virtual enterprise / collaborative networks paradigm to energy networks can be found in [13].

\section{Water Management}

Similarly to energy, water is one of the most important natural resources that need to be preserved and adequately managed. Since fresh water sustains life and is a finite and vulnerable resource, effective management of water resources demands a holistic approach, linking social and economic development with protection of natural ecosystems.

One particular aspect is the management of water distribution networks at regional level. In many cases those networks are managed by public entities, often associated with local city and town governments. In some countries, there is a trend to the privatization of these services. Then a new actor becomes critical, acting as the regulator or supervising authority. In addition, consumers are becoming more 
demanding, requiring from the water supply services very high levels of quality. Quality is not only related to the biological or physical-chemical factors, but also related to the continuity of the supply with adequate levels of pressure and flow. Therefore, the objectives of the water supply managers are not only to deliver water, but to distribute it with the lowest possible cost and within the currently accepted quality levels. In order to cope with variable demands, one water supplier might need to cooperate with neighboring suppliers.

Earlier developments in this area were focused on the development of some forms of intelligent supervision systems in order to reduce costs and guarantee proper quality of service but with an intra-organizational perspective [14]. The development of more advanced management systems however, shall consider an interorganizational perspective to cope with the needed interactions among all stakeholders, e.g. water suppliers, distributors, regulators, local administration, consumers (people, industries), consumer associations, etc. Thus, collaborative networks may provide the adequate framework for this sector.

\section{Biodiversity Infrastructures}

Currently, a wide variety of stakeholder organizations involved in biodiversity activities, e.g. biodiversity data providers, laboratories, universities, conservation groups, etc., are increasingly participating in some form of networked interaction. However, all current networks are goal-oriented, as the main common reason for organizations to involve in their establishment is to share their competencies and resources as required to perform specific tasks which they cannot perform alone. In other words, current collaboration among the Bio-diversity organizations results from the need to obtain / share some resources available at other organizations, including physical resources (e.g. chemical processing machinery and equipments, specialized transportation means, or collected data and samples), human resources (e.g. specialists, researchers, knowledge workers), and/or technological resources (e.g. software systems, processing power), in order to achieve certain common goals. For example, in the collaboration scenario addressed in the FlySafe project [15] which is aimed at protecting airplanes from colliding with migrating birds, several regional Biodiversity-related Organizations which are involved in research on migrating birds, as well as a few external organizations involved in weather forecast, airport administration, etc. are co-working to jointly achieve the aim of reducing risks for the airplanes.

Nevertheless the future plans for Biodiversity virtual labs in Europe as well as worldwide, is stepping out of the current networking framework and aiming to support a distributed research infrastructure, well-equipped to explore, describe, predict and propose decisive actions, as well as impacting the state of biodiversity for the benefit of the society. To achieve these goals however, the pre-existence of a VBE, namely a strategic collaborative biodiversity network is the antecedent to: (i) the acquisition and management of the vast amount of existing and emergent dispersed data/information, (ii) the required diverse processing and integration of this data, as well as (iii) the development of new methods and software systems required for modelling the complexity, testing, and decision making. In this direction, the 
vision addressed within the LifeWatch European Infrastructure initiative [16], for instance, addresses the need for the development of global networks and infrastructures to support the activities of biodiversity stakeholders. To achieve this aim, all organizations active and/or related to different biodiversity domains shall participate in sustainable strategic networks, which will act as the Breeding environments for the formation of Temporary Collaborative Networks (BTCNs). The BTCNs are long term strategic virtual organizations, the so-called VBEs [17], which aim to equip and engage the entire biodiversity community in cooperating, coworking, and co-development. Once the BTCNs are established, goal-oriented Temporary Collaborative Networks (TCNs) may be configured to fulfil emerging society/market opportunities. With the support of their BTCNs, the TCNs embed the potential to cope with required unpredictable changes, as well as collaborating with other European and global initiatives.

The main potential benefits foreseen to be offered to the BOs through the BTCNs include: (i) Getting recognised as a stakeholder in the biodiversity domain, through the label of the corresponding BTCN community, (ii) Access to elements of the BTCN's Bag of Assets (e.g. shared data, pointers to relevant free software, some shared proprietary software, lessons learned, shared "procedures" to perform experiments, publication library), (iii) Benefiting from the BTCN branding, and public relation, and having a voice influencing policy makers, (iv) Provision of common effective ICT-infrastructures for interoperability and collaboration, (v) Increasing the chances of TCN involvement, even from remote geographic regions, through the BTCN management mechanisms, and (vi) Provision of mechanisms, guidelines, and assisting services to motivate/facilitate TCN establishment.

In order to comprehensively specify the BTCN characteristics for the biodiversity domain, the ARCON reference modelling framework for Collaborative Networks [5] is being applied in the LifeWatch initiative.

\section{Collaborative Networks and Ageing}

One important element of sustainability under the social pillar is the demographic dimension, leading to the term "demographic sustainability". This is usually understood as a constant population level related to an optimal growth rate of the population, corresponding to at least 2.1 fertility rate [18]. Another perspective of demographic sustainability is given by the relationship between working age and older population. Current demographic trends are leading to a growing unbalance, which has a large impact on the economic subsystem. Particularly, the ageing population increases the pressure on the state pension funds. In the design of most social security pension schemes pensioners' benefits are paid by those who are currently working. With relatively fewer people working and greater numbers claiming pensions, the long term sustainability of this "intergenerational contract" is in danger.

Recently there is a growing recognition that the elderly population should not be considered a burden on the society but instead an asset that needs to be properly considered. A critical challenge for the society [19] is to identify new organizational structures, approaches, and mechanisms so that elderly citizens do not feel excluded, 
and have the chance to use their knowledge and expertise in making valued contributions to the communities where they live. In particular the senior professionals / knowledge workers possess a number of skills and accumulated experience that need to be used better in value creation activities. As a support element, ICT, and particularly high-speed pervasive broadband connectivity and webbased technologies, offer new opportunities to create new ways of working, facilitate social interaction, and reduce limitations imposed by location and time, thus increasing personal control.

Currently, associations of retired professionals represent an important organizational structure supporting active ageing. It is however important to avoid that such associations turn into a ghetto for seniors. Their effective involvement in the socio-economic system needs to consider other stakeholders, namely intermediary entities and the recipients of the services provided by seniors. Some of these entities are also organized in some form of association e.g. associations of SMEs, enterprise incubators or science parks, networks of regional development agencies and associated private (small) consultancy companies. Therefore a tripartite network model (Fig. 2) for these collaborative networks [20] is a promising approach.

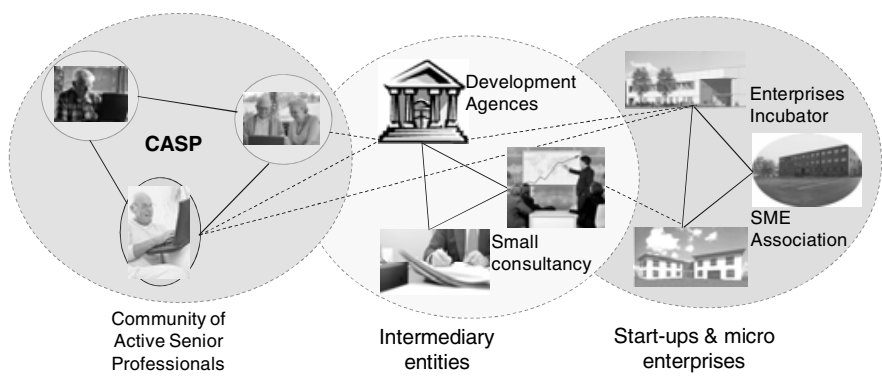

Fig. 2. Tri-partite networks in extending active professional life for seniors

Another case for the application of the $\mathrm{CN}$ approach is the provision of care for elderly. An integrated elderly care system consists of a number of organizations such as care centers, health care institutions, social security institutions, emergency services, and involves the collaboration of a number of different human actors e.g. social care assistants, health care professionals, the elderly people, and their relatives [21], [22].When based on computer networks and adequate supporting tools, collaboration among care institutions may be organized to operate as a long-term virtual organization and the various involved humans become part of a virtual community. In this direction, the ePAL and BRAID projects have been elaborating strategic research roadmaps for ICT and ageing.

\section{Crime Networks}

Criminal networks may constitute an important obstacle to sustainability, at least from the social pillar perspective. In some regions, there is even the risk of some countries being "taken over" and controlled by criminal networks. 
Although this area of research is still at its early stages, as identified by Hernández [23], collaborative networks theory and models can be a useful instrument in refining the current understanding of criminal networks, helping to understanding their evolution, and ultimately providing hints regarding new approaches to fight them. For instance, in his pioneering work, Hernández uses the concepts of virtual organization and VO Breeding Environment to analyze Colombia's criminal networks. Approaches and mechanisms put forth by Social Network Analysis are also being used to analyze and visualize the structure of criminal networks, as in the work of $\mathrm{Xu}$ and Chen [24].

A complementary perspective in the area of criminality is to use network analysis to improve the performance of terrorism defense organizations. Policy chains constitute complex organizations having to coordinate one with another to develop, implement and administrate control public policies. Characterized by a large number of autonomous actors, policy chains often struggle with non-transparency among organizations (lack of overview on the actors involved, lack of information exchange, biases in the collaboration induced by relative power relationships, etc.) and, thus, with coordination weaknesses. To improve policy chain performance, Boorst \& al [25] propose to use a network analysis method [26] to settle performance metrics adapted to terrorism defense organizations.

On the other hand, the study of these organizations, for instance understanding their resilience, could bring new insights for better management and the survival of business networks in times of market turbulence.

\section{Sustainable Tourism}

According to the World Tourism Organization, sustainable tourism development initiatives should meet the needs and interests of current tourists flowing within a country while at the same time ensuring the protection of the environment and enhancement of tourism opportunities for the future generations. Particularly, tourism in developing countries represents a unique case as it is suffering from a number of bottlenecks. For example [27]:

- excessive dependency on foreign investment and companies, contributing to a loss of local control over resources and substantial overseas leakage of tourism earnings;

- a lack of articulation between touristic enclaves and domestic economic sectors, producing low multiplier and spread effects;

- a large-scale environmental destruction, often involving nonrenewable resources and foundation assets;

- a rising alienation among the local population of host communities due to the unequal distribution of the costs and benefits of tourism and the perceived loss of cultural identity and social control to outsiders.

Such social as well as economic problems call for sustainable tourism initiatives, which stress on a wide-ranged involvement of tourism stakeholders, especially SMEs and government institutions, through collaborative networks. Early applications of collaborative networks to tourism (e.g. [28]), were mostly focused on the composition of multi-provider value-added services. More recent initiatives [29] address the role 
of $\mathrm{CNs}$ in the environmental governance for sustainable tourism. From a governance perspective two main types of networks are emerging [29]:

- Policy planning networks, which result from the need for cooperation and collaboration in tourism planning. Public institutions are the main players, as those defining priorities and policies. However, an increasing number of entities from the private sector are now being involved.

- Self-regulating action networks, based on action-oriented practices towards environment sustainability. Such networks involve distinct organizations sharing the same purposes, within the same territorial unit, including private businesses as well as non-governmental associations, aiming at creating mutual opportunities (a kind of business ecosystem).

For this sector, there is the need to manage all resources in such a way that economic, social and cultural needs can be fulfilled while maintaining cultural integrity, essential ecological processes, biological diversity, and life support systems. The complex interplay of the social, cultural and environmental dimensions of sustainable tourism requires a broad understanding and acceptance from different stakeholders, working directly and indirectly in the tourism sector. Therefore, the engagement of the key stakeholders e.g. accommodation providers, tour operators, leisure industry, catering companies, governmental and cultural entities, brokers and traveling agencies, etc., into a collaborative business ecosystem can create conditions for better sharing and more conscious use of resources.

\section{Disaster Rescue Networks}

Situations of disaster or crisis recovery constitute typical examples of complex collaborative networks which have been attracting increasing attention from the scientific community in the last few years [30], [31]. A crisis (being human induced or triggered by the forces of nature), is a complex and dynamic phenomenon, it is characterized by a high level of collective threat and risk, with a great need of reactivity throughout the crisis duration and a constant necessity of adaptation from the part of the recovery organisation. Such constraints, as well as the multiplicity of organisational and institutional actors to be coordinated during the management of a crisis, raise many challenges for collaboration. Efficacy and efficiency of collaborative crisis management require a sustainable approach: disaster events have a strong social impact and reducing this impact thanks to a very reactive response appears essential; in many cases, such crises have direct environmental consequences; the economic dimension is generally linked to management of the financial means allocated to disaster rescue. As a rather recent field of application for collaborative networks, this area of research quickly evolves both by integrating the theoretical knowledge now available on collaborative networks as well as by developing an indepth analysis of the collaboration specificities in the context of crisis and disasters.

Lack of coordination and failures in collaboration and interoperability have often been pointed out as key issues to improve crisis management, in the case of humanitarian disasters [32] but also for other types of crisis [33]. Much research work is currently trying to provide a better understanding of the nature of coordination and collaboration in 
such contexts, as a first step before defining specifically adapted solutions. At a high level, collaboration starts with the coordination of intentionality among the various stakeholders. Organisational proximities integrate various notions of cognitive, social, cultural or institutional proximities which have to be considered as collaboration facilitators [34]. For instance, such factors can designate long-term humanitarian clusters, as means to improve the agile implementation of collaborative networks when a crisis occurs. This example puts forth that an in-depth knowledge on this specific context (i.e. organisational proximities) has to be developed before applying more generic concepts of collaborative science (i.e. Virtual Breeding Environment).

Heterogeneity among stakeholders' intentionality and rationality has significant impact on the coordination of decision processes. Performance management systems primarily based on improving coordination among stakeholders are a first way to increase decisional efficiency. Until now, for crises context, performance evaluation has been mainly used after crisis settlement, notably because of a lack of trustable indicators [35]. To make possible a broader use of performance management as a support of the crisis response process, specific performance indicators systems are required, to take into account the particularity of disaster contexts [36]. Typically, in the field of humanitarian supply chains, generic and reusable indicator systems are proposed as a reference to facilitate the development of more specific applications. If current advances already integrate the sociological and economic aspects of crisis management, the integration of environmental indicators is still a perspective for further works.

Disaster management, perhaps even more than any other type of virtual organisation, underlines the crucial information technology challenges to support collaboration. Crisis situations constitute strongly dynamic environments where the adaptability of information systems to the changes over time of the collaboration processes appears crucial [37]. Model-driven approaches for information system implementation provide a conceptual framework to support dynamic and adaptable collaborative information systems. For instance, considering crisis as an evolutionary process, the ISyCri project has developed a Mediation Information System to support the dynamic interoperability among changing stakeholders [33]. Supporting the evolutionary features of virtual recovery organisations with reactive information and decision systems remains a key challenge for future work.

\section{Conclusions}

Pursuing sustainable solutions is one of today's major challenges for the society. The need to consider the three main pillars of sustainability calls for both a more holistic perspective of the problems and a tighter collaboration among a wide range of stakeholders. Collaborative networks can bring a significant contribution to the better understanding of the stakes and paths towards potential solutions. As illustrated, there are already a large number of examples, in different domains, where $\mathrm{CN}$ contributions to the sustainability agenda are identified and new approaches are being tried. A continuation of this effort is likely to raise new research challenges and to ultimately help us building more sustainable systems.

Acknowledgments. Partial support for this work was given by the European Commission through the ePAL and BRAID projects. 


\section{References}

1. Kates, R., Parris, T., Leiserowitz, A.: What is Sustainable Development? Environment 47(3), 821 (2005), http://www.hks.harvard. edu/sustsci/ists/docs/whatissD _env_kates_0504.pdf

2. United Nations General Assembly: Report of the World Commission on Environment and Development: Our Common Future. Transmitted to the General Assembly as an Annex to document A/42/427 - Development and International Co-operation: Environment (1987), http: / / www . un-documents . net/wced-ocf .htm

3. United Nations General Assembly: 2005 World Summit Outcome, Resolution A/60/1, adopted by the General Assembly on September 15 (2005)

4. Adams, W.M.: The Future of Sustainability: Re-thinking Environment and Development in the Twenty-first Century. Report of the IUCN Renowned Thinkers Meeting, January 29-31 (2006), http: / / cmsdata.iucn.org/downloads/iucn_future_of_sustanability. paf

5. Camarinha-Matos, L.M., Afsarmanesh, H.: Collaborative Networks: Reference Modeling. Springer, Heidelberg (2008)

6. Bojar, W.L., Drelichowski, L.: Analysis of tendencies in agribusiness networking coopetition in Poland and in the partner countries. Journal of Central European Agriculture 9(3), 445-456 (2008)

7. Volpentesta, A.P., Ammirato, S.: Networking agrifood SMEs and consumer groups in local agribusiness. In: Pervasive Collaborative Networks, pp. 33-40. Springer, Heidelberg (2008)

8. Camarinha-Matos, L.M., Carelli, R., Pellicer, J., Martin, M.: Towards the virtual enterprise in food industry. In: Re-Engineering for Sustainable Industrial Production, pp. 73-84. Chapman \& Hall, Boca Raton (1997)

9. FORAGRO: Agriculture and rural prosperity from the perspective of technological research and innovation in LAC: FORAGRO Position 2010. Forum of the Americas for Agricultural Research and Technological Development (March 2010)

10. Osório, A.L., Camarinha-Matos, L.M., Gomes, J.S.: A collaborative case study: The extended "ViaVerde" toll payment system. In: Proceedings of PRO-VE 2005 - Collaborative Networks and their Breeding Environments, pp. 559-568. Springer, Heidelberg (2005)

11. Breuer, W., Povh, D., Retzmann, D., Urbanke, C., Weinhold, M.: Prospects of Smart Grid Technologies for a Sustainable and Secure Power Supply. In: $20^{\text {th }}$ World Energy Congress, Rome, Italy, November 11-15 (2007)

12. Kok, J.K., Warmer, C.J., Karnouskos, S., Nestle, D., Dimeas, A., Weidlich, A., Strauss, P., Buchholz, B., Drenkard, S., Hatziargyriou, N., Lioliou, V.: Smart houses for a smart grid. In: 20th International Conference on Electricity Distribution, Prague, Czech Republic, June 8-11 (2009)

13. Allan, L., Menzel, K.: Virtual Enterprises for Integrated Energy Service Provision. In: Leveraging Knowledge for Innovation in Collaborative Networks, pp. 659-666. Springer, Heidelberg (2009)

14. Afsarmanesh, H., Camarinha-Matos, L.M.: Federated knowledge integration and machine learning in water distribution networks. In: Proc. ISIP 1997 Int. Conf. on Integrated and Sustainable Industrial Production, Re-Engineering for Sustainable Industrial Production, Lisbon, Portugal, pp. 121-140. Chapman \& Hall, Boca Raton (May 1997)

15. FlySafe FP6 project, http: / / www. eu-flysafe. org / Project. html (viewed 2008-11-03)

16. LifeWatch - e-science and technology infrastructure for biodiversity data and observatories, http: / / www. Iifewatch.eu/

17. Afsarmanesh, H., Camarinha-Matos, L.M.: A framework for management of virtual organizations breeding environments. In: Proceedings of 6th PRO-VE 2005 - Collaborative Networks and their Breeding Environments, pp. 35-48. Springer, Heidelberg (2005) 
18. Roca, M.N.O., Leitão, N.: Demographic Sustainability In Portugal: Towards a Typology. In: 14th European Colloquium on Theoretical and Quantitative Geography, Tomar, Portugal (2005)

19. Camarinha-Matos, L.M., Afsarmanesh, H.: Collaborative mechanisms for a new perspective on active ageing. In: Proceedings of DEST 2009 - 3rd IEEE Int. Conference on Digital Ecosystems and Technologies, Istanbul, Turkey, June 1-3 (2009)

20. Camarinha-Matos, L.M., Afsarmanesh, H., Ferrada, F.: Collaborative Networks Approach to Active Ageing. In: Proc. of Pervasive Health 2010 / AGEmap workshop, Munich, Germany, March 22-25 (2010)

21. Camarinha-Matos, L.M., Afsarmanesh, H.: TeleCARE: Collaborative virtual elderly care support communities. The Journal on Information Technology in Healthcare 2(2), 73-86 (2004) ISSN 1479-649X

22. Camarinha-Matos, L.M., Afsarmanesh, H.: A multi-agent based infrastructure to support virtual communities in elderly care. Int. J. Networking and Virtual Organisations 2(3), 246-266 (2004) ISSN 1470-9503

23. Hernández, A.: Comparing Notes: Collaborative Networks, Breeding Environments, and Organized Crime. In: Leveraging Knowledge for Innovation in Collaborative Networks. IFIP AICT, vol. 307, pp. 87-95. Springer, Heidelberg (2009)

24. $\mathrm{Xu}, \mathrm{J}$., Chen, H.: Criminal Network Analysis and Visualization. Communications of the ACM 48(6), 101-107 (2005)

25. Baaijens, J.M.J., Lapré, L.J.: Network analysis of policy chains in the Netherlands. In: 20th EGOS Colloquium, Ljubljana (July 2004)

26. Borst, I., Baaijens, J.M.J., Meijer, G.R.: Network analysis of terrorism defense organizations - A network approach for developing performance indicators. In: Proceedings of PRO-VE 2005, Virtual Enterprise and Collaborative Network, Valencia, Spain, September 26-28, pp. 265-272 (2005)

27. Robertson, P.J.: An Assessment of Collaborative Governance in a Network for Sustainable Tourism: The Case of RedeTuris. In: 10th National Public Management Research Conference (2009)

28. Afsarmanesh, H., Camarinha-Matos, L.M.: Future smart organizations: A virtual tourism enterprise. In: Proceedings of WISE $2000-1^{\text {st }}$ ACM/IEEE International Conference on Web Information Systems Engineering, June 19-20. Main Program, vol. 1, pp. 456-461. IEEE Computer Society Press, Hong Kong (2000)

29. Erkus-Ozturk, H., Eraydın, A.: Environmental governance for sustainable tourism development: Collaborative networks and organisation building in the Antalya tourism region. Tourism Management 31, 113-124 (2010)

30. Devlin, E.: Crisis Management Planning and Execution. Auerbach Publications (2006)

31. Kovács, G., Spens, K.: Humanitarian Logistics in Disaster Relief Operations. International Journal of Physical Distribution and Logistics Management 37, 99-114 (2007)

32. Beamon, B.M., Balcik, B.: Performance measurement in humanitarian relief chains. Int. J. Public Sect. Manag. 21(1), 4-25 (2008)

33. Truptil, S., Bénaben, F., Couget, P., Lauras, M., Chapurlat, V., Pingaud, H.: Interoperability of Information Systems in Crisis Management: Crisis Modeling and Metamodeling. In: IESA 2008. Springer, Germany (2008)

34. Hyypiä, M., Kautonen, M.: Dimensions of Proximity in Relationships between Knowledge Intensive Business Service Firms and Their Clients. In: Regional Studies Association International Conference on Regional Growth Agendas, Aalborg, May 28-31 (2005)

35. Kovács, G., Tatham, P.H.: What is special about a humanitarian logistician? A survey of logistic skills and performance. Supply Chain Forum - An International Journal (2004)

36. Balcik, B.: Relief chain planning and management: modeling and analyzing humanitarian logistic problems. PhD thesis, University of Washington (2008)

37. Oloruntoba, R., Gray, R.: Humanitarian aid: an agile supply chain? Supply Chain Management: An International Journal 11, 115-120 (2006) 